\title{
Dog color patterns explained by modular promoters of ancient canid origin
}

2 Danika L. Bannasch*1,2, Christopher B. Kaelin*3,4, Anna Letko ${ }^{2,5}$, Robert Loechel ${ }^{6}$, Petra Hug ${ }^{2,5}$,

3 Vidhya Jagannathan ${ }^{2,5}$, Jan Henkel ${ }^{2,5}$, Petra Roosje ${ }^{5,7}$, Marjo K. Hytönen ${ }^{8,9,10}$, Hannes Lohi ${ }^{8,9,10}$,

4 Meharji Arumilli ${ }^{8,9,10}$, DoGA consortium, Katie M. Minor ${ }^{11}$, James R. Mickelson ${ }^{11}$, Cord

$6{ }^{1}$ Department of Population Health and Reproduction, School of Veterinary Medicine, University

7 of California Davis, Davis, CA 95616 USA.

$8{ }^{2}$ Institute of Genetics, Vetsuisse Faculty, University of Bern, 3001, Bern, Switzerland.

$9 \quad{ }^{3}$ HudsonAlpha Institute for Biotechnology, Huntsville, AL, USA.

$10{ }^{4}$ Department of Genetics, Stanford University, Stanford, CA, USA.

$11{ }^{5}$ Dermfocus, University of Bern, 3001 Bern, Switzerland.

$12{ }^{6}$ VetGen, Ann Arbor, MI, 48108, USA.

$13{ }^{7}$ Division of Clinical Dermatology, Department of Clinical Veterinary Medicine, Vetsuisse

14 Faculty, University of Bern, 3001 Bern, Switzerland.

$15{ }^{8}$ Department of Veterinary Biosciences, University of Helsinki, 00014 Helsinki, Finland.

$16{ }^{9}$ Department of Medical and Clinical Genetics, University of Helsinki, 00014 Helsinki, Finland.

$17 \quad{ }^{10}$ Folkhälsan Research Center, 00290 Helsinki, Finland.

${ }^{11}$ Department of Veterinary and Biomedical Sciences, University of Minnesota, Saint Paul, MN 55108, USA.

$20 *$ \& These authors contributed equally to this work 
and after domestication from wolves ${ }^{1,2}$ but important gaps remain in understanding how

these patterns evolved and are genetically controlled ${ }^{3,4}$. In other mammals, variation at the ASIP gene controls both the temporal and spatial distribution of yellow and black pigments 3,5-7. Here we identify independent regulatory modules for ventral and hair cycle $A S I P$ expression, and we characterize their action and evolutionary origin. Structural variants define multiple alleles for each regulatory module and are combined in different ways to explain five distinctive dog color patterns. Phylogenetic analysis reveals that the haplotype combination for one of these patterns is shared with arctic white wolves and that its hair cycle-specific module likely originated from an extinct canid that diverged from grey wolves more than 2 million years before present. Natural selection for a lighter coat during the Pleistocene provided the genetic framework for widespread color variation in dogs and wolves.

A central aspect of the amazing morphologic diversity among domestic dogs are their colors and color patterns. In many mammals, specific color patterns arise through differential regulation of Agouti $(A S I P)$, which encodes a paracrine signaling molecule that causes hair follicle melanocytes to switch from making eumelanin (black or brown pigment) to pheomelanin

41 (yellow to nearly white pigment) ${ }^{8}$. In laboratory mice, Asip expression is controlled by alternative promoters in specific body regions, and at specific times during hair growth, and gives rise to the light-bellied agouti phenotype, with ventral hair that is yellow and dorsal hair that contains a mixture of black and yellow pigment ${ }^{7}$. Genetic variation in ASIP affects color pattern in many mammals; however, in dogs, the situation is still unresolved, in large part due to the complexity of different pattern types, and challenges in distinguishing whether genetic 
investigate non-coding variation in ASIP regulatory modules and their effect on patterning phenotypes in domestic dogs. We expand our analysis to include modern and ancient wild canids and uncover an evolutionary history in which natural selection during the Pleistocene provided a molecular substrate for color pattern diversity today. with a yellow color are dominant to those associated with a black color. Although dominant
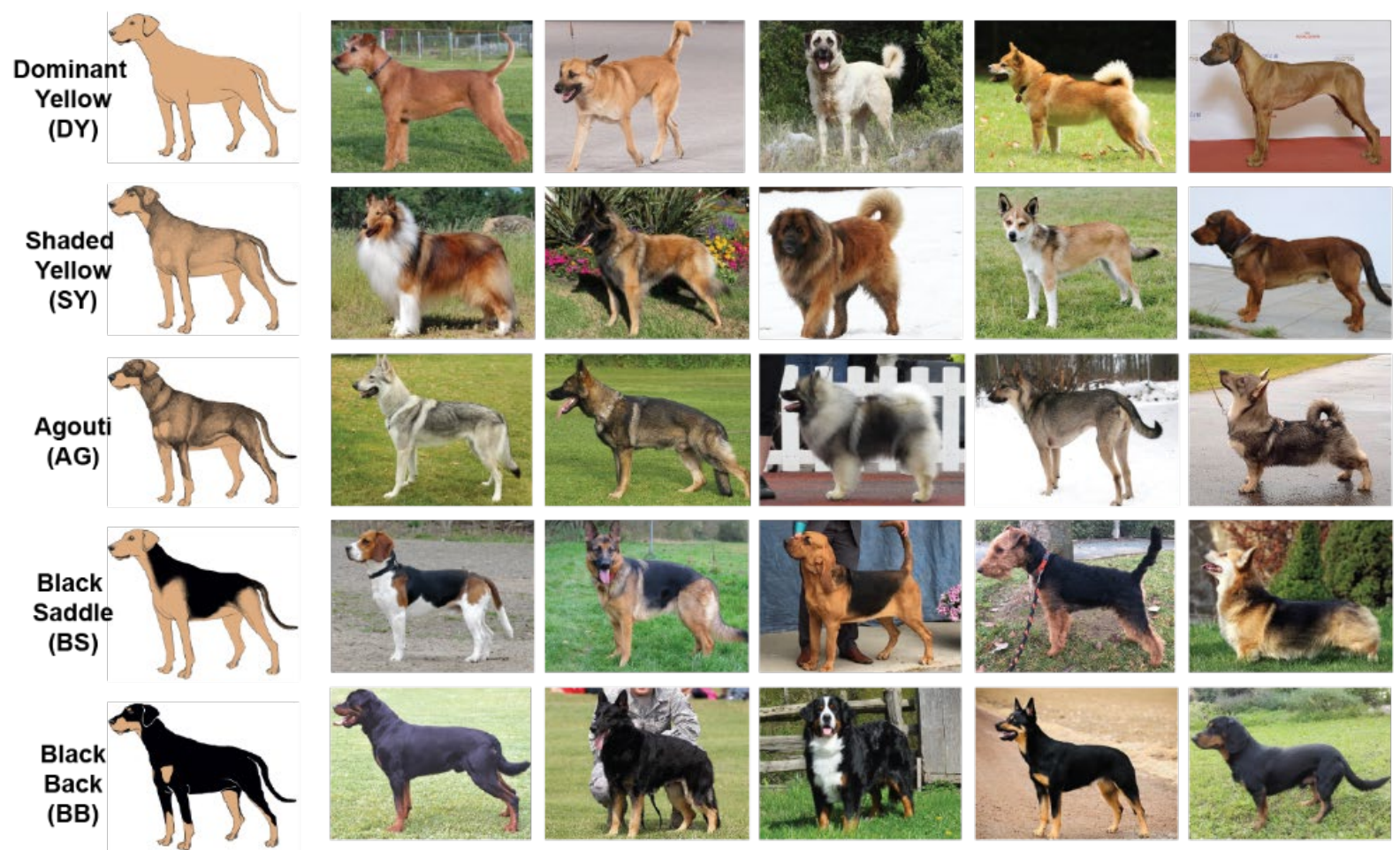
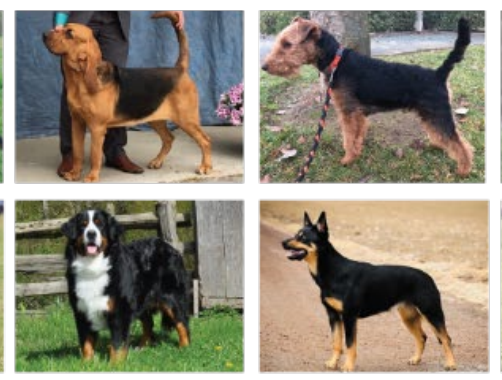

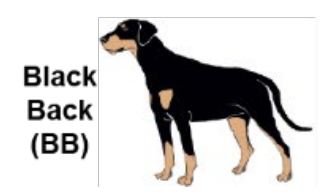


61 Fig. 1: Coat patterns controlled by the $\boldsymbol{A S I P}$ locus. The five phenotype names proposed here

62 are shown on the left. To the right are photographs of representative dogs of various morphological types. Length and curl of hair coat, shade of pheomelanin (red to nearly white),

64 presence of a black facial mask and white spotting are the result of genetic variation at other loci.

65 Patterns are displayed in order of dominance. A completely black coat caused by ASIP loss-of-

66 function (recessive black) is not shown.

67

We collected skin RNA-seq data from dogs with different pattern phenotypes and identified three alternative untranslated first exons for $\operatorname{dog} A S I P$ (Fig. 2a, Supplementary Table activity and characteristic non-coding sequence variation according to dog pattern phenotype.

72 These two promoters are orthologous to the ventral promoter (VP) and hair cycle promoter 73 (HCP) in the laboratory mouse ${ }^{7}$; however, our genetic analyses (Fig. 2) reveal that the dog VP 74 and HCP exhibit greater regional and quantitative variation than their mouse counterparts. inspected whole genome sequence data from 77 dog and wolf samples with known color patterns

77 (Supplementary Table 3). We identified multiple structural variants that lie within $2 \mathrm{~kb}$ of the VP or HCP transcriptional start sites, confirmed their presence and identity by Sanger sequencing, and used homozygous individuals to infer two VP haplotypes and five HCP haplotypes. VP1

80 contains an upstream SINE element and an A-rich expansion not found in VP2 (Fig. 2a, left,

81 Supplementary Table 1); the five HCP haplotypes differ according to the number and identity of 82 upstream SINE elements, as well as additional insertions and deletions (Fig. 2a, right, 
These results were extended by developing PCR-based genotyping assays for the VP and

85 HCP structural variants, examining their association with different pattern phenotypes in 352

86 dogs from 34 breeds, and comparing these results to previously published variants (Extended

87 Data Fig. 2-3, Extended Data Table 1, Supplementary Tables 4-7). As depicted in Fig. 2b,

88 combinations of VP1 or VP2 with $\mathrm{HCP} 1,2,3,4$, or 5 are correlated perfectly with variation in

89 ASIP pattern phenotype. Because the level of ASIP activity is directly related to the amount of

90 yellow pigment production, these genetic association results suggest that VP1 has greater activity

91 than VP2, HCP1 has greater activity than HCP2, and HCP3, 4, and 5 all represent loss-of-

92 function; indeed, the HCP4 haplotype includes a large deletion that includes the hair cycle first

93 exon (Fig. 2a). For example, homozygotes for VP1-HCP1, VP2-HCP1, VP2-HCP2 are dominant

94 yellow, shaded yellow and agouti, respectively (Extended Data Table 1, Supplementary Tables

95 4-7). Black saddle dogs are VP1-HCP4 homozygotes and most black back dogs are VP2-HCP3

96 homozygotes (although all three loss of function HCP haplotypes paired with VP2 can produce

97 the black back phenotype) (Extended Data Fig.3 and Supplementary Table 7). Increased activity

98 from the ventral promoter (VP1 vs. VP2) correlates with dorsal expansion of yellow pigment in

99 black saddle compared to black back phenotypes (Fig. 1, 2b), which indicates that the VP and

100 HCP haplotypes function separately from each other. 
a

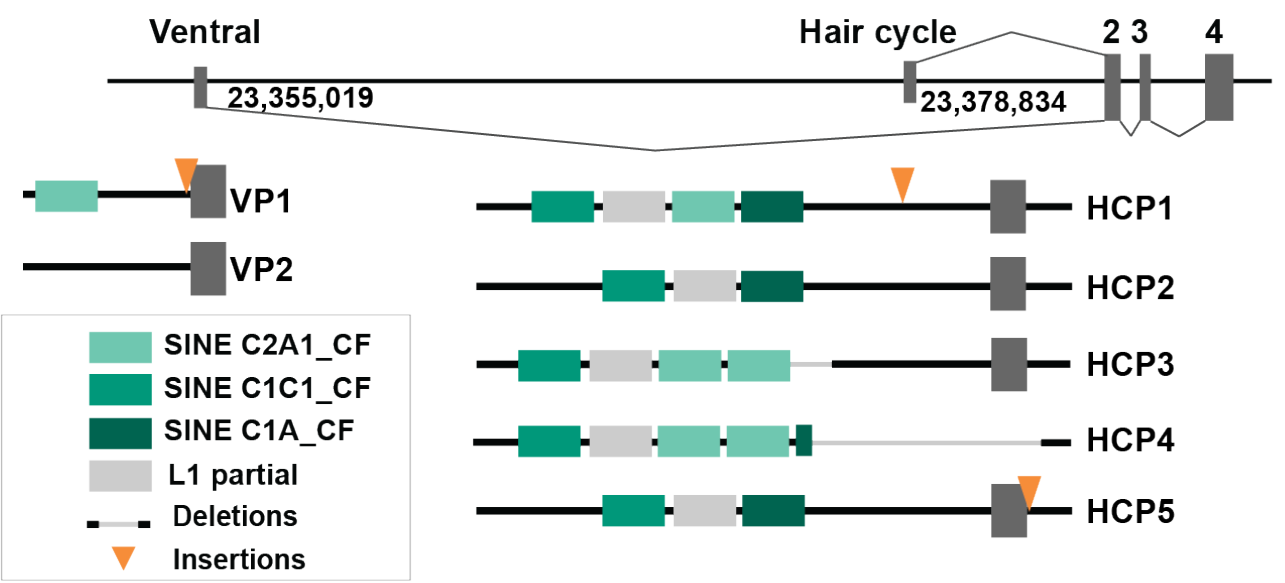

b
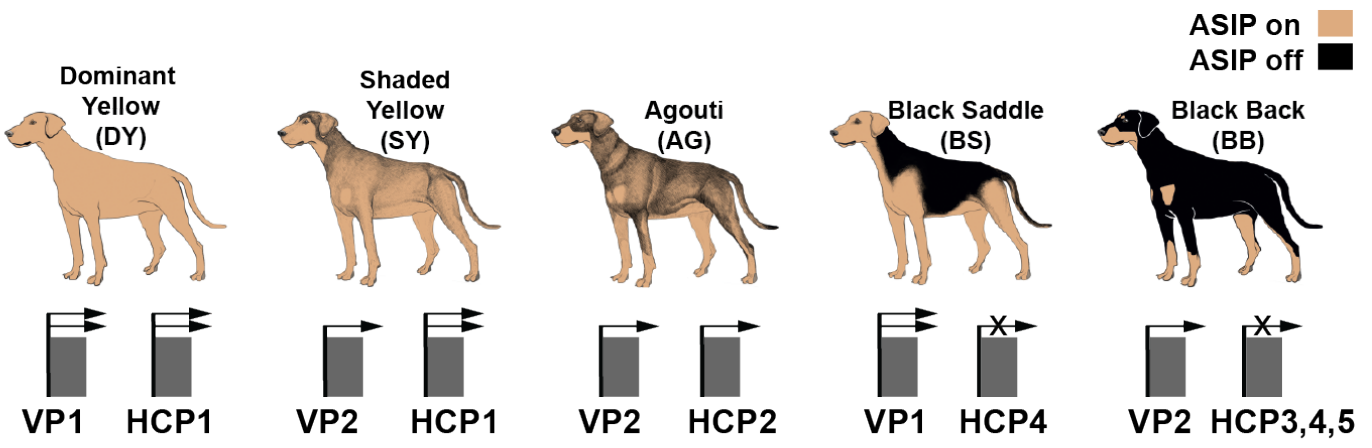

$N=113$

$\mathrm{N}=51$

$\mathrm{N}=46$

$\mathrm{N}=53$

$\mathrm{N}=89$

Fig. 2: Structural variation at the $A S I P$ locus in domestic dogs with different color patterns

(a) Schematic of the two relevant alternative transcription start sites and first exons (nucleotide coordinates denote their 3'-ends), together with the haplotypes observed. (b) Summary of how extended haplotype combinations are related to color pattern phenotypes. Semi-quantitative expression levels are depicted with one or two arrows or an $\mathrm{X}$ for no expression (Extended Data Fig. 1). $\mathrm{N}$ is the number of dogs for which the VP and HCP haplotype combinations accounted for $A S I P$ pattern phenotype. An additional 14 dogs had a dark mask (due to an $M C 1 R$ variant) which prevented accurate assignment of ASIP pattern phenotype (Extended Data Table 1, Supplementary Tables 4-7). 

explored further using biopsies of dorsal and ventral skin (Supplementary Table 8, Extended

114 Data Fig. 1). Read counts from RNA-seq data were consistent with expectations from the genetic

115 association results: VP1 has greater transcriptional activity and is spatially broadened relative to

116 VP2 (which is only expressed ventrally), HCP1 has greater transcriptional activity relative to

$117 \mathrm{HCP} 2$, and no reads are detected from HCP3 or HCP4 (Fig. 2B, Extended Data Fig. 1). Taken

118 together, these results provide a molecular explanation for ASIP pattern variation in dogs in

119 which the VP and HCP haplotypes function as independent regulatory modules for their

120 associated promoters and first exons.

122 comparing haplotypes in 18 homozygous dogs to those from 10 contemporary grey wolves (Fig.

123 3a, Supplementary Table 9). Overall, agouti dog haplotypes were similar to those from grey

124 wolves. However, dominant yellow and, to a lesser extent, shaded yellow dog haplotypes were

125 similar to those from arctic grey wolves from Ellesmere Island and Greenland, where all wolves

126 are white (Fig. 3a, 3c). Notably, white coat color in wolves represents pale pheomelanin, as in

127 Kermode bears or snowshoe hares ${ }^{10,11}$. In the $64 \mathrm{~kb}$ segment that contains the VP, HCP, and

128 coding sequence, the arctic grey wolf haplotypes are identical except for one polymorphic site,

129 and are distinguished from dog dominant yellow haplotypes by only 6 SNVs (Extended Data

130 Table 2). Taken together, these observations suggest a common origin of dominant yellow in

131 dogs and white coat color in wolves without recent genetic exchange. 


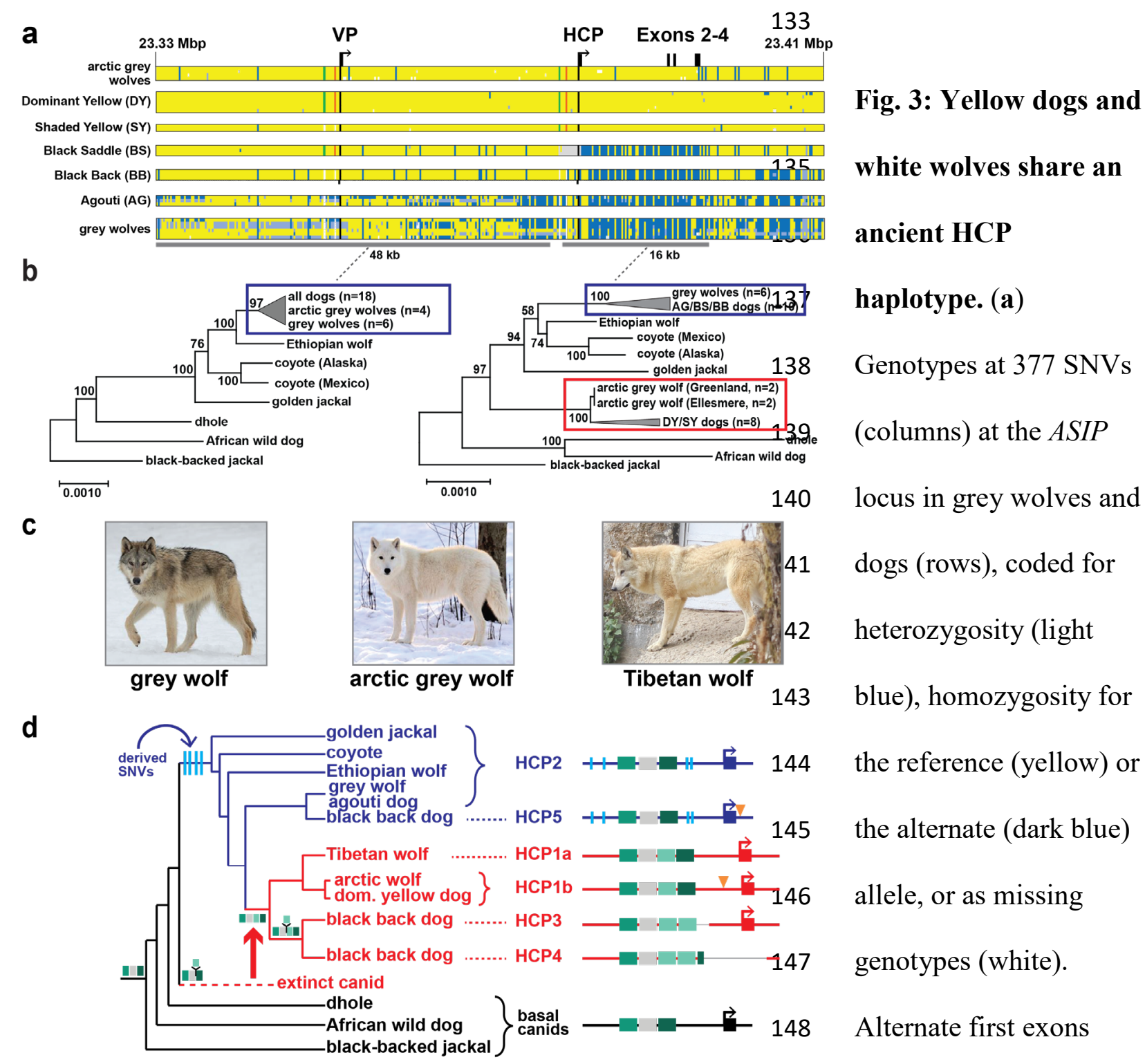

149 (arrows) and nearby DY-associated structural variants (SINE insertions: green, polynucleotide

150 expansions: orange) are included for reference. (b) Maximum likelihood phylogenies, including

151 seven extant canid species and the dog, from 48 and $16 \mathrm{~kb}$ intervals upstream or downstream of

152 the HCP, respectively. Grey wolf/dog phyletic clades are highlighted with boxes to indicate

153 relationships that are consistent (blue) or inconsistent (red) with genome-wide phylogenies. (c)

154 Images of a grey wolf, arctic grey wolf, Tibetan wolf. (d) A phylogeny representing distinct HCP

155 evolutionary histories inferred from genetic variation in extant canids. Structural variants (as 
represented in Fig. 2) and derived SNVs (cyan) distinguish wolf-like canid (blue), ghost lineage (red), and basal canid (black) haplotypes.

The evolutionary origin of $A S I P$ haplotypes was explored further by constructing maximum likelihood phylogenetic trees for dogs, wolves, and 8 additional canid species (Supplementary Table 9). Based on differences in SNV frequency, the $48 \mathrm{~kb}$ VP segment was considered separately from the $16 \mathrm{~kb}$ HCP-exon 2/3/4 segment (see supplementary text, Fig. 3a). In the VP tree, all dogs and grey wolves form a single clade, consistent with known species relationships ${ }^{12}$. However, in the HCP tree, the dominant yellow and shaded yellow dogs lie in a separate clade together with arctic grey wolves; remarkably, this clade is basal to the golden jackal and distinct from other canid species (Fig. 3b, Extended Data Fig. 4, 5).

The pattern of derived allele sharing provides additional insight (Fig. 3d and Extended Data Fig. 6). As depicted in Fig. 2b and 3d, HCP2 is characterized by three small repeat elements that are shared by all canids and is therefore the ancestral form. In the branch leading to core wolf-like canids (golden jackal, coyote, Ethiopian wolf, and grey wolf), there are nine derived SNV alleles within the HCP2-exon 2/3/4 segment (Extended Data Fig. 6), four of which flank the repeat elements close to HCP2 (Fig. 3d). None of the nine derived alleles are present in the dominant yellow HCP1-exon 2/3/4 segment haplotype (which also carries an additional SINE close to $\mathrm{HCP} 1$; therefore this haplotype must have originated prior to the last common ancestor of golden jackals and other wolf-like canids $>2$ Mybp ${ }^{13}$. Although the $16 \mathrm{~kb}$ HCP1-exon 2/3/4 segment haplotype could have originated on a branch leading to the core wolf-like canids, it would have had to persist via incomplete lineage sorting and absence of recombination for more than 2 million years and through three speciation events (supplementary text). A more likely scenario is that HCP1 represents a ghost lineage from an extinct canid (Fig. 3d, 4b) that was 
179 introduced by hybridization with grey wolves during the Pleistocene (see below), as has been

180 suggested for an ancestor of the grey wolf and coyote ${ }^{12}$, and in high altitude Tibetan and

181 Himalayan wolves ${ }^{14}$.

We expanded our analysis of VP and HCP haplotypes to a total of 45 North American extend to exon $2 / 3 / 4$ and lacks the $24 \mathrm{bp}$ insertion found in arctic grey wolves and dominant yellow dogs (Supplementary Table 10). The Tibetan and arctic grey wolf haplotypes are referred to as HCP1a and HCP1b, respectively (Fig. 3d, Extended Data Fig. 7, 8). The VP1-HCP1b haplotype combination is found mostly in the North American Arctic in a distribution parallel to that of white coat color (Extended Data Fig. 7a) ${ }^{15}$. This haplotype combination is not observed 189 in Eurasia, although one similar to shaded yellow, VP2-HCP1a, was observed in seven lightcolored wolves from Tibet or Inner Mongolia (Fig. 3d, Extended Data Fig. 7b) ${ }^{16}$.

196 River areas of Arctic Siberia had at least one HCP1 haplotype, while ancient dogs from central

197 Europe, Ireland, and Siberia carried HCP1a, HCP1b, and HCP4, respectively (Supplementary

198 Table 10). Thus, diversity in $A S I P$ regulatory sequences responsible for color variation today was 


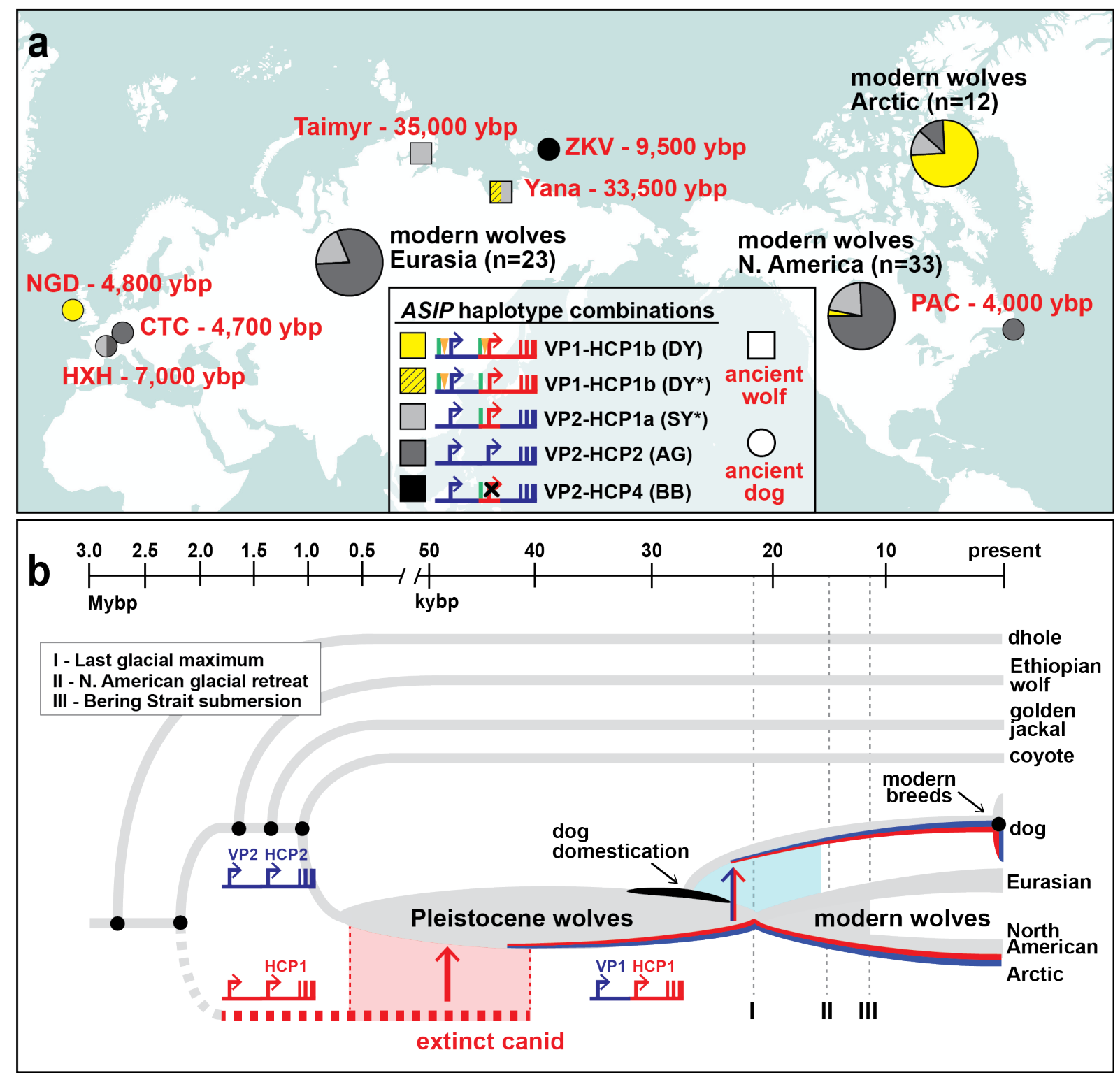

Fig. 4: Distribution of $A S I P$ alleles in ancient dogs and wolves, and an evolutionary model

for dominant yellow acquisition. (a) $A S I P$ haplotypes were inferred from whole genome charts) distributed across the Holarctic (see Supplementary Table 10 and Extended Data Fig. 8 for detailed haplotype representations). Asterisks indicate SY/DY haplotypes for which the HCP1 insertion is either absent (SY*) or not ascertainable (DY*). (b) A model for origin of the dominant yellow haplotype and its transmission into dogs and arctic wolves, in which molecular 
208

209

210

211

212

213

214

215

216

217

218

219

220

221

222

223

224

225

226

227

228

229

alterations at modular promoters were acquired by introgression (red, HCP1) or by mutation in the grey wolf (blue, VP1). The timeline for speciation events, dog domestication, and geological events affecting grey wolf dispersal are based on prior studies ${ }^{13,17}$.

Together with our phylogenetic results, comparative analysis of wolf and dog ASIP haplotypes suggests an evolutionary history in which multiple derivative haplotypes and associated color patterns arose by recombination and mutation from two ancestral configurations corresponding to a white wolf (VP1-HCP1) and a grey wolf (VP2-HCP2), both present in the late Pleistocene (Fig. 4a, Extended Data Fig. 8). The distribution of derivative haplotypes explains color pattern diversity not only in dogs but also in modern wolf populations across the Holarctic, including white wolves in the North American Arctic (VP1- HCP1b) and yellow wolves in the Tibetan highlands (VP2-HCP1a), and is consistent with natural selection for light coat color.

A likely timeline for the origin of modules driving high levels of $A S I P$ expression is depicted in Fig. $4 \mathrm{~b}$ and indicates a dual origin. The HCP1 haplotype represents introgression into Pleistocene grey wolves from an extinct canid lineage that diverged from grey wolves more than 2 Mybp. This introgression as well as the mutation from VP2 to VP1 occurred prior to 33,500 ybp, based on direct observation from an ancient wolf sample (Fig. 4a). Natural selection for VP1 and HCP1 are a likely consequence of Pleistocene adaptation to arctic environments and genetic exchange in glacial refugia, driven by canid and megafaunal dispersal during interglacial periods. Modern grey wolves are thought to have arisen from a single source $\sim 25,000$ ybp close to the last glacial maximum ${ }^{18,19}$; during the North American glacial retreat that followed, the VP1-HCP1b haplotype combination was selected for in today's white-colored arctic wolves. 
In dogs, ASIP color pattern diversification was likely an early event during

231 domestication, since our analysis of ancient DNA data reveals several different VP and HCP

232 haplotypes in Eurasia by 4,800 ybp. This is consistent with the wide distribution of dominant

233 yellow across modern dog breeds from diverse locations, as well as the dingo (Supplementary

234 Table 9), a feral domesticate introduced to Australia at least 3,500 ybp ${ }^{20}$. Of particular interest is

235 the Zhokov island dog from Siberia ${ }^{21,22}$. Based on a haplotype combination of VP2-HCP4, this

236 sled dog that lived 9,500 years ago exhibited a black back color pattern, allowing it to be easily

237 distinguished from white colored wolves in an arctic environment. Our results show how

238 introgression, demographic history, and the genetic legacy of extinct canids played key roles in

239 shaping this diversity.

240 References

2411 Linderholm, A. \& Larson, G. The role of humans in facilitating and sustaining coat

242 colour variation in domestic animals. Semin Cell Dev Biol 24, 587-593,

243 doi:10.1016/j.semcdb.2013.03.015 (2013).

2442 Wayne, R. K. \& vonHoldt, B. M. Evolutionary genomics of dog domestication. Mamm

245 Genome 23, 3-18, doi:10.1007/s00335-011-9386-7 (2012).

2463 Caro, T. \& Mallarino, R. Coloration in Mammals. Trends Ecol Evol 35, 357-366,

247 doi:10.1016/j.tree.2019.12.008 (2020).

2484 Dreger, D. L. et al. Atypical Genotypes for Canine Agouti Signaling Protein Suggest

249 Novel Chromosomal Rearrangement. Genes (Basel) 11, doi:10.3390/genes11070739 (2020).

2505 Henkel, J. et al. Selection signatures in goats reveal copy number variants underlying

251 breed-defining coat color phenotypes. PLoS Genet 15, e1008536, 
2536 Linnen, C. R. et al. Adaptive evolution of multiple traits through multiple mutations at a

254 single gene. Science 339, 1312-1316, doi:10.1126/science.1233213 (2013).

2557 Vrieling, H., Duhl, D. M., Millar, S. E., Miller, K. A. \& Barsh, G. S. Differences in

256 dorsal and ventral pigmentation result from regional expression of the mouse agouti gene. Proc

257 Natl Acad Sci U S A 91, 5667-5671, doi:10.1073/pnas.91.12.5667 (1994).

2588 Barsh, G., Gunn, T., He, L., Schlossman, S. \& Duke-Cohan, J. Biochemical and genetic 259 studies of pigment-type switching. Pigment Cell Res 13 Suppl 8, 48-53, doi:10.1034/j.1600-

$260 \quad 0749.13 . s 8.10 . x(2000)$.

2619 Kaelin, C. B. \& Barsh, G. S. Genetics of pigmentation in dogs and cats. Annu Rev Anim

262 Biosci 1, 125-156, doi:10.1146/annurev-animal-031412-103659 (2013).

26310 Ritland, K., Newton, C. \& Marshall, H. D. Inheritance and population structure of the

264 white-phased "Kermode" black bear. Curr Biol 11, 1468-1472, doi:10.1016/s0960-

265 9822(01)00448-1 (2001).

26611 Jones, M. R. et al. Adaptive introgression underlies polymorphic seasonal camouflage in

267 snowshoe hares. Science 360, 1355-1358, doi:10.1126/science.aar5273 (2018).

26812 Gopalakrishnan, S. et al. Interspecific Gene Flow Shaped the Evolution of the Genus

269 Canis. Curr Biol 28, 3441-3449.e3445, doi:10.1016/j.cub.2018.08.041 (2018).

27013 Koepfli, K. P. et al. Genome-wide Evidence Reveals that African and Eurasian Golden

271 Jackals Are Distinct Species. Curr Biol 25, 2158-2165, doi:10.1016/j.cub.2015.06.060 (2015).

27214 Wang, M. S. et al. Ancient hybridization with an unknown population facilitated high

273 altitude adaptation of canids. Mol Biol Evol, doi:10.1093/molbev/msaa1 13 (2020). 
27415 Gipson PS, E. E. B., Theodore N. Bailey, Diane K. Boyd, H. Dean Cluff, Douglas W.

275 Smith and Michael D. Jiminez Color Patterns among Wolves in Western North America.

276 Wildlife Sociaety Bulletin 30, 821-830 (2002).

27716 Zhang, W. et al. Hypoxia adaptations in the grey wolf (Canis lupus chanco) from

278 Qinghai-Tibet Plateau. PLoS Genet 10, e1004466, doi:10.1371/journal.pgen.1004466 (2014).

27917 Freedman, A. H. \& Wayne, R. K. Deciphering the Origin of Dogs: From Fossils to

280 Genomes. Annu Rev Anim Biosci 5, 281-307, doi:10.1146/annurev-animal-022114-110937

281 (2017).

28218 Fan, Z. et al. Worldwide patterns of genomic variation and admixture in gray wolves.

283 Genome Res 26, 163-173, doi:10.1101/gr.197517.115 (2016).

28419 Loog, L. et al. Ancient DNA suggests modern wolves trace their origin to a Late

285 Pleistocene expansion from Beringia. Mol Ecol, doi:10.1111/mec.15329 (2019).

28620 Balme, J., O'Connor, S. \& Fallon, S. New dates on dingo bones from Madura Cave

287 provide oldest firm evidence for arrival of the species in Australia. Sci Rep 8, 9933,

288 doi:10.1038/s41598-018-28324-x (2018).

28921 Lee, E. J. et al. Ancient DNA analysis of the oldest canid species from the Siberian

290 Arctic and genetic contribution to the domestic dog. PLoS One 10, e0125759,

291 doi:10.1371/journal.pone.0125759 (2015).

29222 Sinding, M.-H. S. et al. Arctic-adapted dogs emerged at the Pleistocene-Holocene

293 transition. Science 368, 1495-1499, doi:10.1126/science.aaz8599 (2020).

29423 Dreger, D. L., Parker, H. G., Ostrander, E. A. \& Schmutz, S. M. Identification of a

295 mutation that is associated with the saddle tan and black-and-tan phenotypes in Basset Hounds

296 and Pembroke Welsh Corgis. J Hered 104, 399-406, doi:10.1093/jhered/est012 (2013). 
29724 Dreger, D. L. \& Schmutz, S. M. A SINE insertion causes the black-and-tan and saddle

298 tan phenotypes in domestic dogs. J Hered 102 Suppl 1, S11-18, doi:10.1093/jhered/esr042

299 (2011).

30025 Berryere, T. G., Kerns, J. A., Barsh, G. S. \& Schmutz, S. M. Association of an Agouti

301 allele with fawn or sable coat color in domestic dogs. Mamm Genome 16, 262-272,

302 doi:10.1007/s00335-004-2445-6 (2005).

303

\section{Methods}

All data generated or analyzed during this study are included in this published article (and its

306

307

308

309

310

311

312

313

314

315

316

317

318

319 supplementary information files).

\section{Ethics Statement}

All animal experiments were done in accordance with the local regulations. Experiments were approved by the "Cantonal Committee For Animal Experiments" (Canton of Bern; permits $48 / 13,75 / 16$ and $71 / 19)$.

\section{Skin biopsies and total RNA extraction}

Skin biopsies were taken from three dogs (a black back Miniature Pinscher and a dominant yellow Border Terrier and Irish Terrier). Two $6 \mathrm{~mm}$ punch biopsies were taken from differentially pigmented body areas of each animal (dorsal and ventral). RNA samples from dogs represent asynchronous hair growth relative to the hair cycle. The biopsies were immediately put in RNAlater (Qiagen) for at least $24 \mathrm{~h}$ and then frozen at $-20^{\circ} \mathrm{C}$. Prior to RNA extraction, the skin biopsies were homogenized mechanically with the TissueLyser II device from Qiagen. Total RNA was extracted from the homogenized tissue using the RNeasy Fibrous Tissue Mini Kit (Qiagen) according to the manufacturer's instructions. RNA quality was assessed with a 
320 FragmentAnalyzer (Agilent) and the concentration was measured using a Qubit Fluorometer

321 (ThermoFisher Scientific).

322 Whole transcriptome sequencing (RNA-seq)

323 From each sample, $1 \mu \mathrm{g}$ of high quality total RNA (RIN $>9$ ) was used for library

324 preparation with the Illumina TruSeq Stranded mRNA kit. The libraries were pooled and

325 sequenced on an S1 flow cell with 2x50 bp paired-end sequencing using an Illumina NovaSeq

3266000 instrument. On average, 31.5 million paired-end reads per sample were collected. One

327 publicly available Beagle sample was used (SRX1884098). All reads that passed quality control

328 were mapped to the CanFam3.1 reference genome assembly using STAR aligner (version 2.6.0c)

32926.

330 Transcript coordinates

331 The STAR-aligned bam files were visualized in the IGV browser ${ }^{27}$. Three different

332 alternate untranslated first exons that appeared to splice to the coding exons of $A S I P$ were

333 defined based on the visualizations of the read alignments in IGV. These exact transcripts have

334 not been documented in NCBI and Ensembl gene models. The visually curated gene models are

335 given in Supplementary Table 2.

336 Identification of genomic variants

337 WGS data from 71 dogs and 6 wolves was used for variant discovery (Supplementary Table

338 3). They included 15 agouti dogs and wolves, 25 black back dogs, 11 black saddle dogs, 14

339 dominant yellow dogs and 11 shaded yellow dogs and one white wolf. The genomes were either

340 publicly available or sequenced as part of related projects in our group ${ }^{28}$. SNVs and small indels

341 were called as described ${ }^{28}$. The IGV software ${ }^{27}$ was used for visual inspection of the promoter 
342 regions based on the transcripts identified in the RNA sequencing data. Structural variants were

343 identified and association with coat color phenotypes was verified by visual inspection in IGV.

DNA samples for Sanger sequencing and genotyping

Samples for variant discovery included two dogs from each color phenotype and are

Table 5 were used for genotyping. The coat color phenotype of all animals was assigned based isolated from EDTA blood samples using the Maxwell RSC Whole Blood DNA kit (Promega).

\section{0}

351

352

353

354

355

356

357

\section{Sequencing of promoter regions}

Sanger sequencing of PCR amplicons was carried out to validate and characterize structural variants at the sequence level in the promoter regions. All primer sequences and polymerases used are listed in Supplementary Table 4. PCR products amplified using LA Taq polymerase (Takara) or Multiplex PCR Kit (Qiagen) were directly sequenced on an ABI 3730 capillary sequencer after treatment with exonuclease I and shrimp alkaline phosphatase. Sequence data were analyzed with Sequencher 5.1 (GeneCodes). Interspersed repeat insertions were classified with the RepeatMasker program ${ }^{29}$. Multiple copies of SINE elements from the same and different families were resolved this way. The CanFam3.1 reference genome assembly is derived from the Boxer Tasha, a dominant yellow dog, and represents a DY haplotype, VP1-HCP1, of the ASIP gene. Descriptions of the promoter variants and Genbank accession numbers for HCP25 are in Supplementary Table 1. The table lists the 7 combinations of VP and HCP regulatory modules observed in dogs. As HCP3, HCP4, and HCP5 all represent loss-of-function alleles that are functionally equivalent, the 7 listed combinations correspond to only 5 distinct phenotypes. 


\section{Genotyping assays}

The previously reported SINE insertion ${ }^{24}$ was genotyped by fragment size analysis on an ABI 3730 capillary sequencer and analyzed with the GeneMapper 4.0 software (Applied

371 (ventral promoter assays 1, 2; hair cycle promoter assays 1, 2, 3) are required to unambiguously

372 determine the VP and HCP haplotypes. The other four primer pairs in the list were used to 373 genotype previously published diagnostic markers ${ }^{23-25}$ or for the amplification of the entire HCP

374 (Supplementary Table 4). Genotyping results for all samples are shown in Supplementary Table

375 5. There is a perfect genotype-phenotype association in 352 dogs (see Fig. 2). In the remaining

37614 dogs, the presence of a eumelanistic mask due to an epistatic $M C 1 R$ allele prevented the

377 reliable phenotypic differentiation of dominant yellow and shaded yellow dogs. Breeds and the

378 different promoter haplotype combinations identified within each breed are indicated in

379 Supplementary Table 6. In a few dogs that were heterozygous at both VP and HCP, the phasing

380 of the VP and HCP haplotype combinations was performed based on haplotype frequency within

381 the same breed as noted. A family of Chinooks were used to determine the segregation of

382 extended haplotypes and the phenotypic equivalency of HCP3 and HCP5 (Extended Data Fig 3).

383 Summary of genotyping results and exclusion of previously associated variants is shown in

384 Supplementary Table 7. This table lists the genotype-phenotype association in aggregated form.

385 The table also contains the genotypes for variants that were previously reported to be associated

386 with pattern phenotypes ${ }^{23-25}$. Numbers in red indicate genotyping results, for which these

387 markers yielded discordant results. 


\section{Comparison of promoter haplotype effects on transcripts}

Transcript data was generated from a second set of samples. Sample descriptions and colors not related to skin. Samples were collected in RNAlater Stabilization Solution and stored at $80^{\circ} \mathrm{C}$. RNA was extracted using the RNeasy Fibrous Tissue Mini Kit (Qiagen) according to manufacturer's instructions. Integrity of RNA was evaluated with Agilent 2100 Bioanalyzer or TapeStation system (Agilent) and concentration measured with DeNovix DS-11 


\section{Haplotype construction}

Haplotypes were constructed from two publicly available VCF files PRJEB32865 and

414 (http://samtools.github.io/bcftools/) with the parameter --missing-to-ref, which assumed

415 genotypes at missing sites are homozygous reference type 0/0. Only dogs homozygous for ASIP

416 haplotypes were used to visualize haplotypes (Supplementary Table 3). SNVs that had 100\% call

417 rate in these samples were color coded and displayed relative to the genome assembly and

418 previously commercialized variants (Extended Data Fig. 2).

\section{ASIP phylogenetic analysis in canids}

Illumina whole genome sequence for 36 canids, including seven extant species and the dog,

were downloaded from the NCBI short read archive as aligned (bam format) or unaligned (fastq

422 format reads (Supplementary Table 9). Fastq data were aligned to the dog genome (CanFam3.1)

423 using BWA (v.0.7.17) ${ }^{34}$ after trimming with Trim Galore (v.0.6.4). SNVs within a $110 \mathrm{~kb}$

424 interval (chr24:23,300,000-23,410,000), which includes the ASIP transcriptional unit and

425 regulatory sequences, were identified with Platypus (v.0.8.1) ${ }^{35}$ and filtered with VCFtools

426 (v.0.1.15) ${ }^{36}$ to include 2008 biallelic SNVs. Phasing was inferred with BEAGLE (v.4.1) ${ }^{37}$.

SNV density (Fig. 3a) and ASIP gene structure: a $48 \mathrm{~kb}$ region including the ventral first exon,

431 (chr24:23,378,001-23,394,000). Consensus sequences of equal length were constructed for each

432 inferred canid haplotype using BCFtools (v.1.9). Phylogenies were inferred using Maximum

433 Likelihood method and Tamura-Nei model with 250 bootstrap replications, implemented in 
434 MEGAX ${ }^{38,39}$, and including 34 canids (Fig. 3b, Extended Data Fig. 4,5). For 34 of 36

435 individuals, consensus haplotype pairs were adjacent to each other or, in the case of a few

436 wolf/dog haplotypes, were positioned in neighboring branches with weak bootstrap support. The

437 exceptions were the African golden wolf, a species derived by recent hybridization of the grey

438 wolf and Ethiopian wolf ${ }^{12}$, and an eastern grey wolf from the Great Lakes region, which was

439 also reported to have recent admixture with the coyote ${ }^{40}$. The African golden wolf and the

440 eastern grey wolf were removed from the alignments, and a single haplotype for each individual

441 was selected arbitrarily for tree building and display.

Haplotype analysis of $A S I P$ locus in ancient dogs and wolves

Whole genome sequencing data from several recent studies ${ }^{12,16,22,41-45}$, including five

444 ancient dogs, two ancient grey wolves, and 68 modern grey wolves (Supplementary Table 10)

445 were downloaded as aligned (bam format) or unaligned (fastq format) reads. Fastq data was

446 aligned to the dog genome (canFam3.1) using BWA-MEM (v.0.7.17) ${ }^{34}$, after trimming

447 withTrim Galore (v.0.6.4). Coverage depth for each sample ranged from 1-78x (Supplementary

448 Table 10). Genotypes at five structural variants and six SNVs were determined by visual

449 inspection using the IGV browser (Supplementary Table 10). Variants in or near the ventral

450 promoter $(n=2)$, the hair cycle promoter $(n=6)$, and the coding exons $(n=3)$ distinguished ventral

451 and hair cycle promoter haplotypes (Supplementary Table 10, Extended Data Fig. 7). SNV

452 genotypes were determined by allele counts; structural variants were genotyped by split reads at

453 breakpoint junctions.

$454 \quad$ For 67 of 75 wolves (or ancient dogs), the phase of ventral and hair cycle promoter

455 haplotypes was unambiguous. Seven wolves and one ancient dog were heterozygous with respect 
456 to both the ventral and hair cycle promoter haplotypes, and for these samples, haplotype phase

457 was inferred based on the linkage disequilibrium in the 67 unambiguous individuals.

458

459

460

461

462

46328 Jagannathan, V., Drogemuller, C., Leeb, T. \& Dog Biomedical Variant Database, C. A

464 comprehensive biomedical variant catalogue based on whole genome sequences of 582 dogs and

465 eight wolves. Anim Genet 50, 695-704, doi:10.1111/age.12834 (2019).

46629 RepeatMasker Open-4.0.

467 (2013).

46830 Islam, S. et al. Characterization of the single-cell transcriptional landscape by highly

469 multiplex RNA-seq. Genome Res 21, 1160-1167, doi:10.1101/gr.110882.110 (2011).

$47031 \quad$ Krjutskov, K. et al. Globin mRNA reduction for whole-blood transcriptome sequencing.

471 Sci Rep 6, 31584, doi:10.1038/srep31584 (2016).

47232 Pertea, M., Kim, D., Pertea, G. M., Leek, J. T. \& Salzberg, S. L. Transcript-level

473 expression analysis of RNA-seq experiments with HISAT, StringTie and Ballgown. Nat Protoc

474 11, 1650-1667, doi:10.1038/nprot.2016.095(2016).

47533 Bray, N. L., Pimentel, H., Melsted, P. \& Pachter, L. Near-optimal probabilistic RNA-seq

476 quantification. Nat Biotechnol 34, 525-527, doi:10.1038/nbt.3519 (2016).

47734 Li, H. \& Durbin, R. Fast and accurate short read alignment with Burrows-Wheeler

478 transform. Bioinformatics 25, 1754-1760, doi:10.1093/bioinformatics/btp324 (2009). 
47935 Rimmer, A. et al. Integrating mapping-, assembly- and haplotype-based approaches for

480 calling variants in clinical sequencing applications. Nat Genet 46, 912-918, doi:10.1038/ng.3036

481 (2014).

48236 Danecek, P. et al. The variant call format and VCFtools. Bioinformatics 27, 2156-2158, 483 doi:10.1093/bioinformatics/btr330 (2011).

48437 Browning, S. R. \& Browning, B. L. Rapid and accurate haplotype phasing and missing485 data inference for whole-genome association studies by use of localized haplotype clustering. $\mathrm{Am}$ 486 J Hum Genet 81, 1084-1097, doi:10.1086/521987 (2007).

48738 Kumar, S., Stecher, G., Li, M., Knyaz, C. \& Tamura, K. MEGA X: Molecular

488 Evolutionary Genetics Analysis across Computing Platforms. Mol Biol Evol 35, 1547-1549, 489 doi:10.1093/molbev/msy096 (2018).

49039 Stecher, G., Tamura, K. \& Kumar, S. Molecular Evolutionary Genetics Analysis

491 (MEGA) for macOS. Mol Biol Evol 37, 1237-1239, doi:10.1093/molbev/msz312 (2020).

$49240 \quad$ vonHoldt, B. M. et al. A genome-wide perspective on the evolutionary history of

493 enigmatic wolf-like canids. Genome Res 21, 1294-1305, doi:10.1101/gr.116301.110 (2011).

49441 Botigué, L. R. et al. Ancient European dog genomes reveal continuity since the Early

495 Neolithic. Nat Commun 8, 16082, doi:10.1038/ncomms16082 (2017).

49642 Frantz, L. A. et al. Genomic and archaeological evidence suggest a dual origin of

497 domestic dogs. Science 352, 1228-1231, doi:10.1126/science.aaf3161 (2016).

49843 Ni Leathlobhair, M. et al. The evolutionary history of dogs in the Americas. Science 361, 499 81-85, doi:10.1126/science.aao4776 (2018). 
50044 Skoglund, P., Ersmark, E., Palkopoulou, E. \& Dalen, L. Ancient wolf genome reveals an

501 early divergence of domestic dog ancestors and admixture into high-latitude breeds. Curr Biol

$502 \quad 25,1515-1519$, doi:10.1016/j.cub.2015.04.019 (2015).

50345 vonHoldt, B. M. et al. Identification of recent hybridization between gray wolves and

504 domesticated dogs by SNP genotyping. Mamm Genome 24, 80-88, doi:10.1007/s00335-012-

$5059432-0$ (2013).

506 Acknowledgements

507 We would like to acknowledge the Next Generation Sequencing Platform of the University of

508 Bern and Biomedicum Functional Genomics Unit (FuGU), University of Helsinki, for

509 sequencing services and the Interfaculty Bioinformatics Unit of the University of Bern and IT

510 Center For Science Ltd. (CSC, Finland) for providing high performance computing

511 infrastructure. We thank resources and members of the Dog Genome Annotation (DoGA)

512 Consortium (Hannes Lohi, Juha Kere, Carsten Daub, Marjo Hytönen, César L. Araujo, Ileana B.

513 Quintero, Kaisa Kyöstilä, Maria Kaukonen, Meharji Arumilli, Milla Salonen, Riika Sarviaho,

514 Julia Niskanen, Sruthi Hundi, Jenni Puurunen, Sini Sulkama, Sini Karjalainen, Antti Sukura,

515 Pernilla Syrjä, Niina Airas, Henna Pekkarinen, Ilona Kareinen, Anna Knuuttila, Heli Nordgren,

516 Karoliina Hagner, Tarja Pääkkönen, Kaarel Krjutskov, Sini Ezer, Shintaro Katayama, Masahito

517 Yoshihara, Auli Saarinen, Abdul Kadir Mukarram, Matthias Hörtenhuber, Amitha Raman, Irene

518 Stevens) as well as the Dog Biomedical Variant Database Consortium and all other canine

519 researchers who deposited genome sequencing data into public databases. We thank the dog

520 owners who provided photographs.

521 


\section{Author Contributions}

524 DB: conceptualization, investigation, writing, visualization, formal analysis, CK: investigation,

525 visualization, formal analysis, writing, AL, PH, RL: validation, resources, VJ and MR: software,

526 PR, JH: validation, KM and JM: resources, MKH, AM, HL, DoGA consortium: resources, STRT

527 analyses, CD: supervision and resources, GB: supervision, writing-review and editing, TL:

528 conceptualization, funding acquisition, investigation, supervision, resources, writing-review and

529 editing.

530 Competing Interest Declaration

531 Authors declare no competing interests except RL who is associated with a commercial

532 laboratory that offers canine genetic testing.

\section{Additional Information}

534 Supplementary Information is available for this paper

535 Correspondence and requests for materials should be addressed to Danika L. Bannasch

536 (dlbannasch@ucdavis.edu)

537 Reprints and permissions information is available at www.nature.com/reprints 


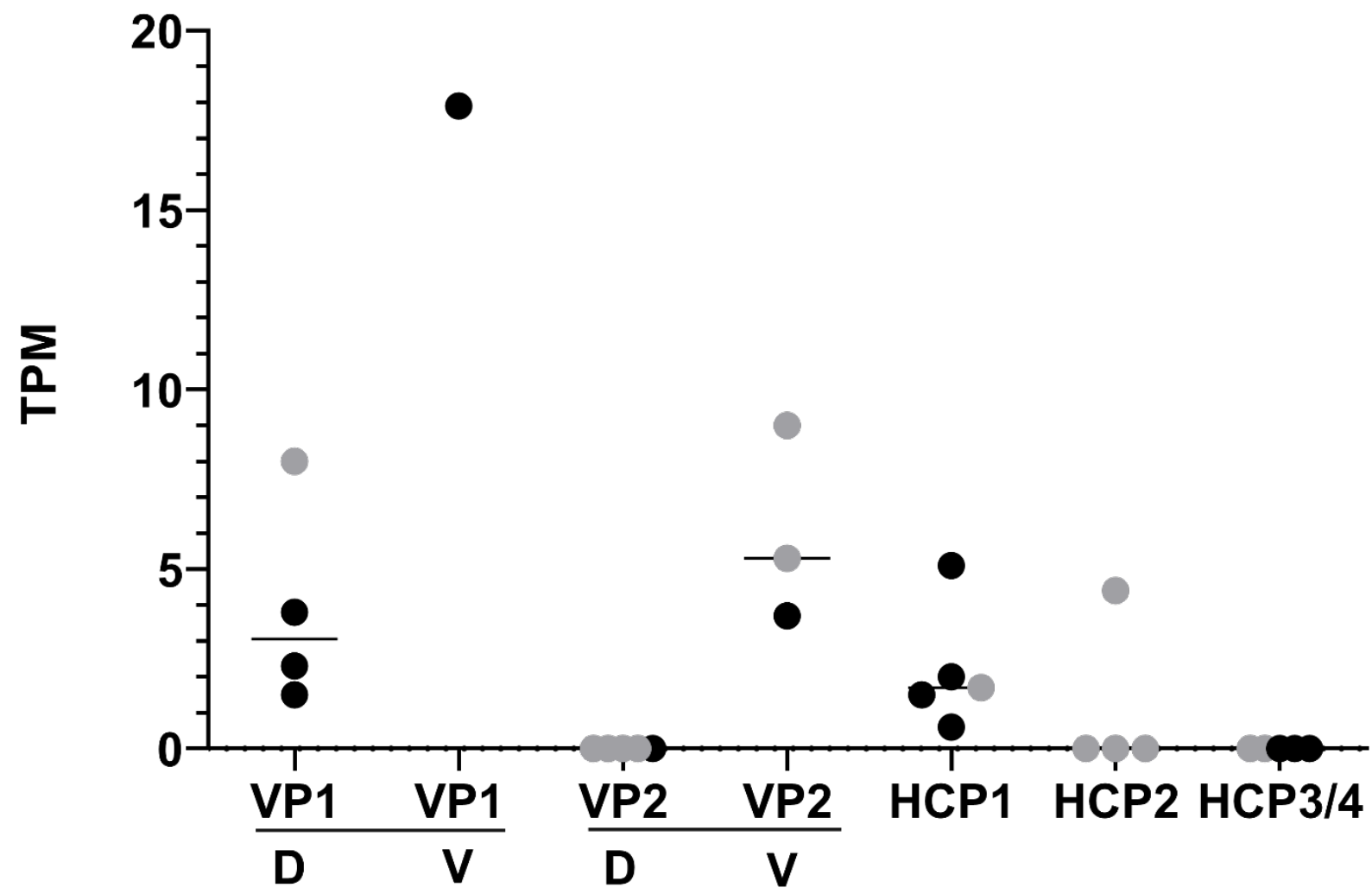

\section{Promoter Haplotype}

547 Extended Data Fig. 1: Relative transcription of promoter variants.

548 Black dots are from RNA-seq data and grey dots are from STRT RNA-seq data.

549 Dorsal samples (D) were taken from mid thorax of the dog and ventral (V) from the ventral

550 abdomen. The HCP samples were not synchronized with respect to the hair cycle. 
552

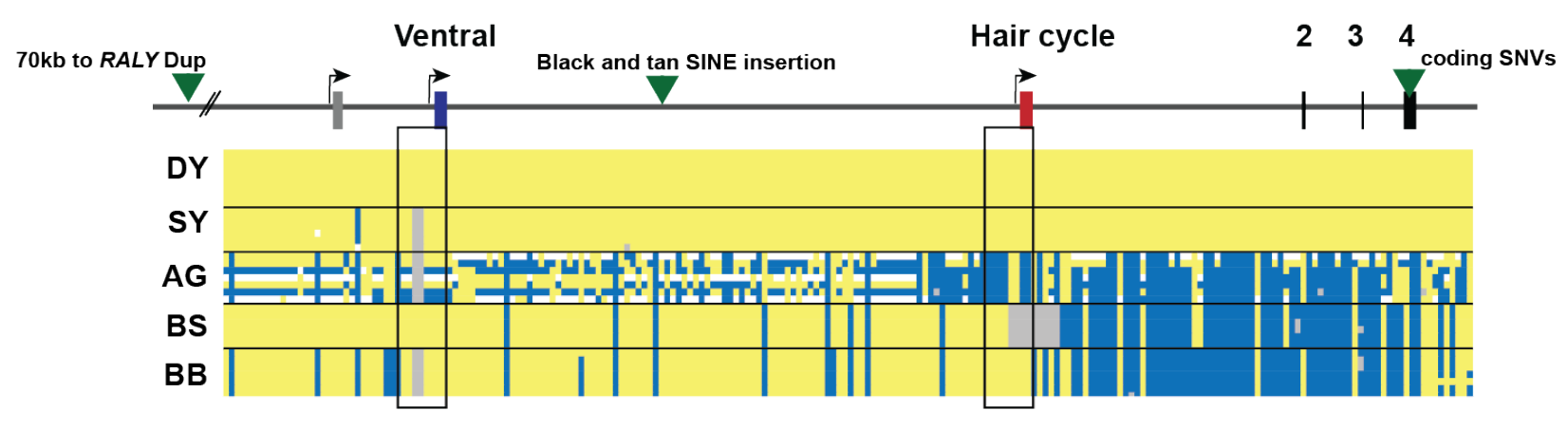

\section{3}

554

Extended Data Fig. 2: Dog haplotypes across the ASIP locus with comparison to

commercial genetic tests for coat color. Dog coat pattern phenotypes are listed on the left.

Alternative first exons are listed at the top. Yellow is a homozygous match to the genome assembly, grey heterozygous, white deleted and blue homozygous alternate allele. The black rectangles highlight the promoter regions. Green triangles represent the location of variants that were previously used in commercial testing to distinguish different alleles for coat color patterns. The previously identified intronic duplication that was promoted to commercially distinguish BS and $\mathrm{BB}$ haplotypes in some breeds lies $70 \mathrm{~kb}$ to the left of this diagram ${ }^{23}$. The green triangle between the VP and HCP is the location of the commercially tested SINE insertion for BB and $\mathrm{BS}^{24}$. In the samples presented here, the dominant yellow haplotype extends through the coding sequence where the missense variants associated with this haplotype were previously identified ${ }^{25}$. In more primitive breeds, recombination events have disrupted this long linkage disequilibrium between the promoter variants and the coding variants leading to incorrect genetic test results with the existing tests. Samples used are listed in Supplementary Table 3. Raw genotyping results are in Supplementary Table 5 and summary results comparing commercial variants are in Supplementary Table 7. 


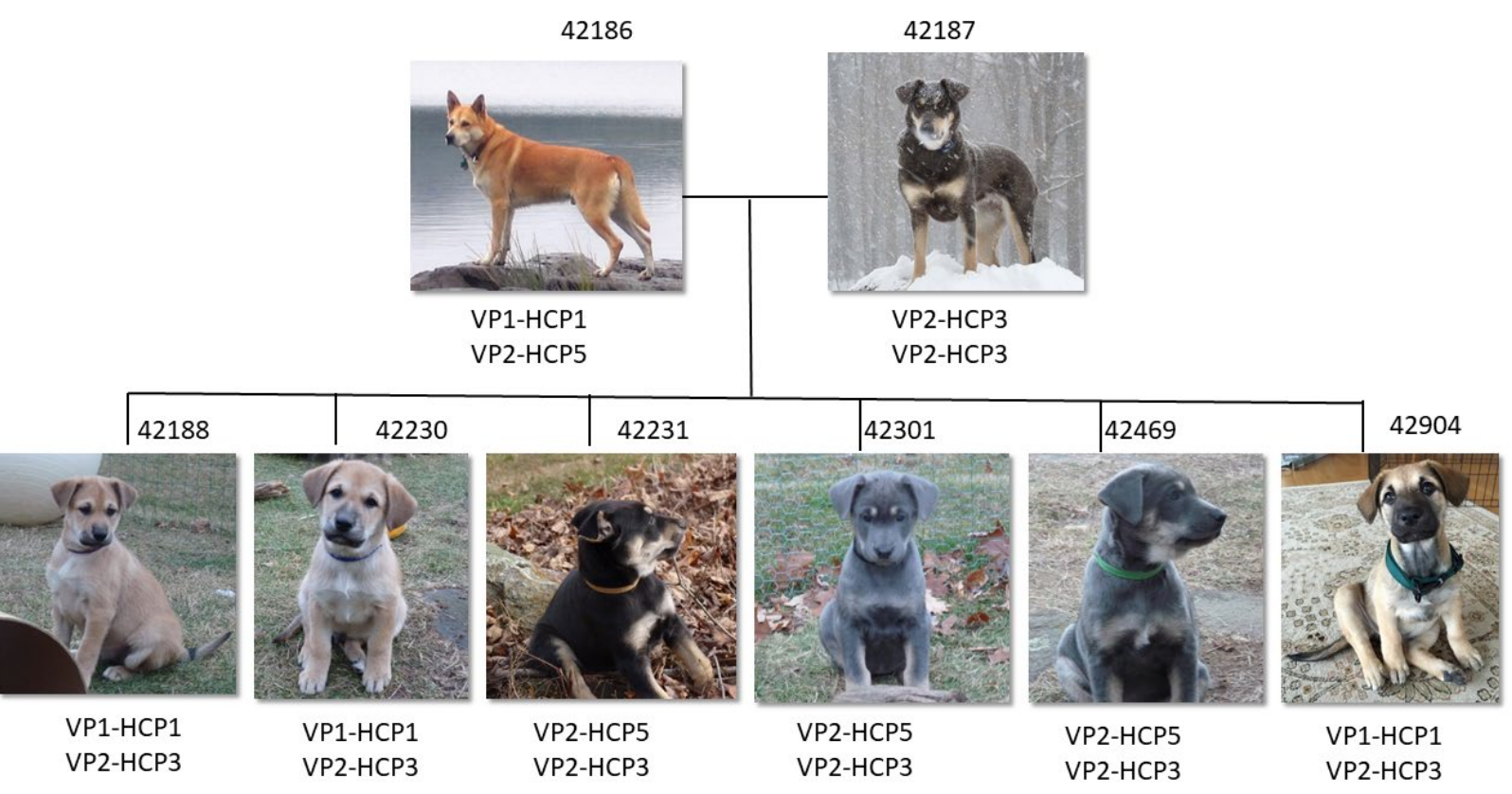

573 Extended Data Fig. 3: A family of dogs segregating dominant yellow and black back.

574 Extended haplotype combinations were determined in this family of Chinook dogs. In this breed

575 both HCP3 and HCP5 segregate and confer a black back phenotype combined with VP2. 


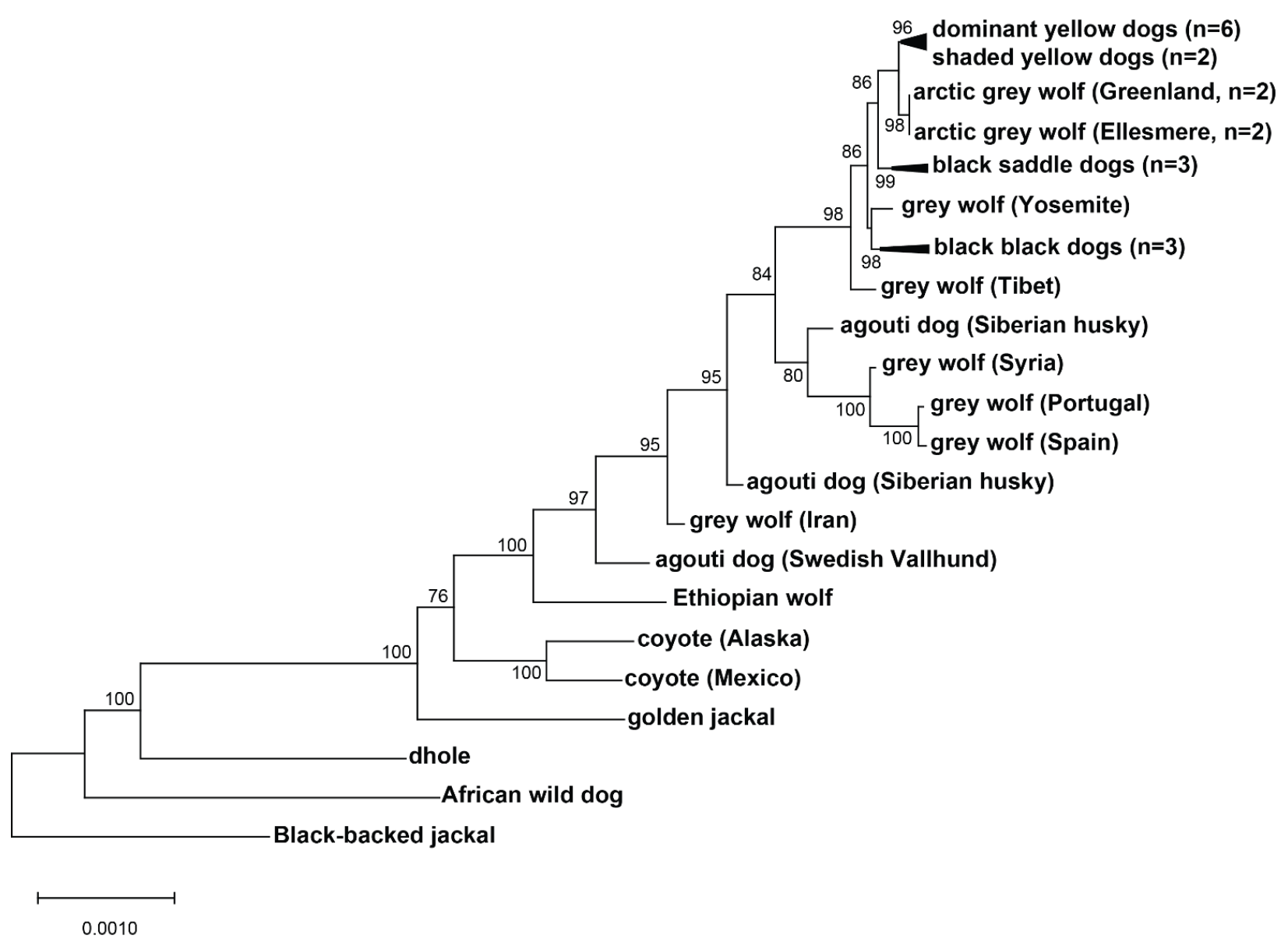

577 Extended Data Fig. 4: Expanded canid phylogenetic tree inferred from 48 kb region

578 including the ventral promoter. An expanded version of the maximum likelihood tree shown in

579 Fig. 3B, with 34 canids, representing 7 of 9 extant species. 


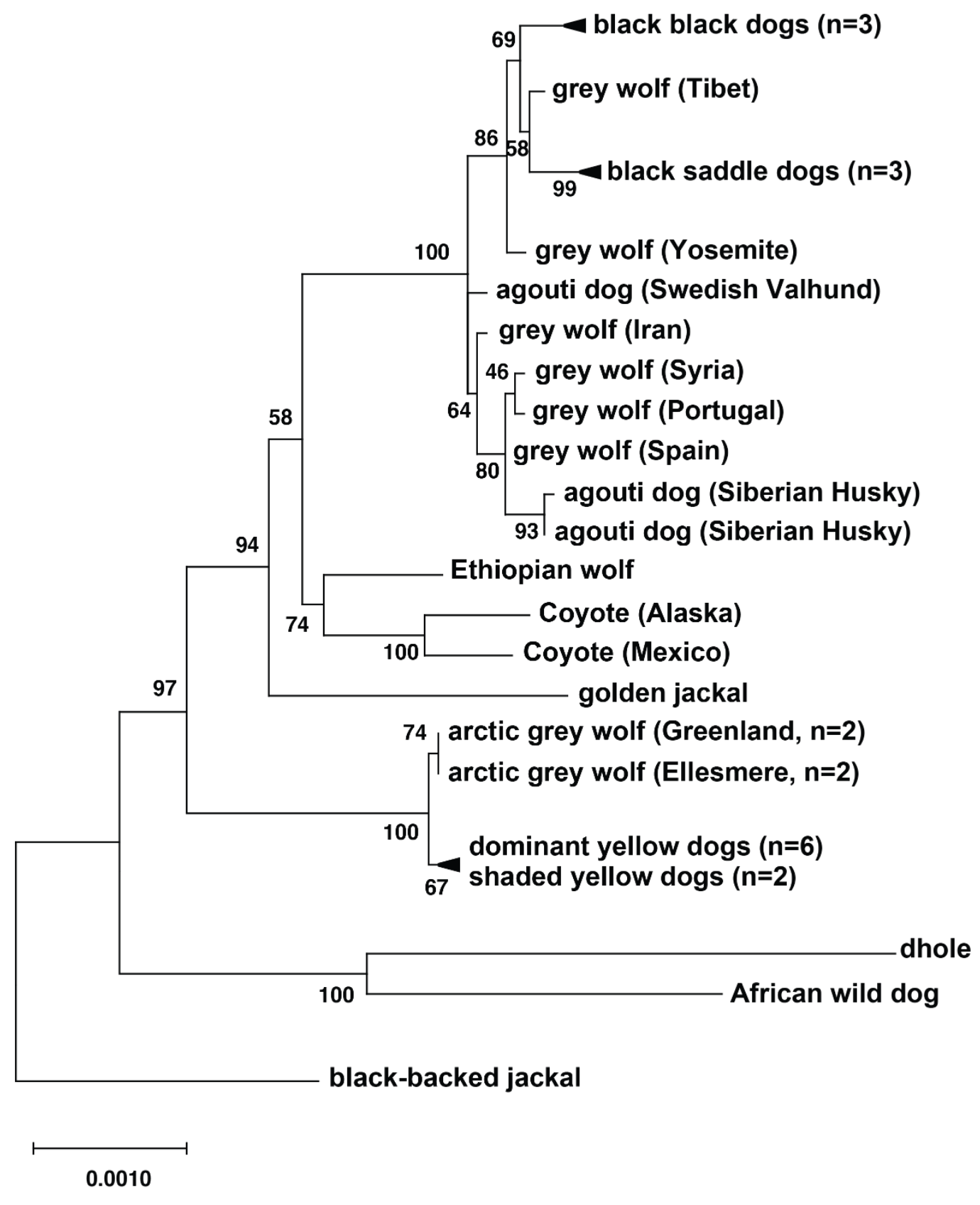

581 Extended Data Fig. 5: Expanded canid phylogenetic tree inferred from 16 kb region within

582 and downstream of the hair cycle promoter. An expanded version of the maximum likelihood 583 tree shown in Fig. 3b, with 34 canids, representing 7 of 9 extant species. 


\section{a $\quad 48 \mathrm{~kb}$ - ventral promoter}

\section{6 kb - hair cycle promoter}
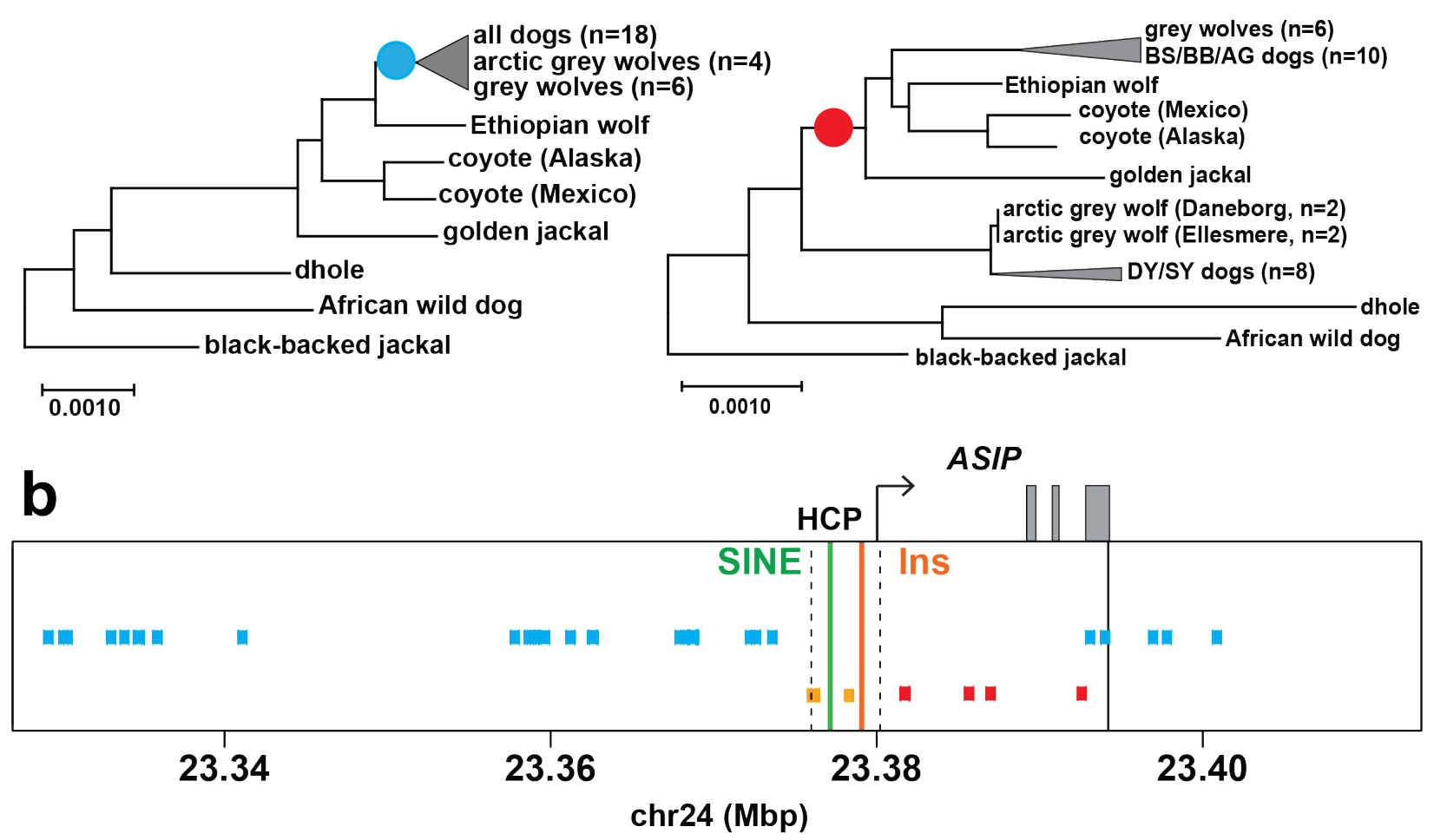

Extended Data Fig. 6: Genomic distribution of derived substitutions across the $A S I P$ locus.

(a) Canid phylogenies for the ventral $(48 \mathrm{~kb})$ and hair cycle $(16 \mathrm{~kb})$ promoter regions, with

relevant internal branches marked by the occurrence of derived variants plotted in (B). (b) haplotypes (red) or BB and DY/arctic wolf haplotypes (orange) that correspond to derived substitutions in the core wolf-like canids (Supplementary Table 11). The broken lines demarcate

592 the HCP region (chr24:23,375,800-23,380,000). The solid line signifies the downstream

593 boundary for phylogenetic analysis. The solid green and orange lines indicate the positions of the

594 SINE and 24 bp insertion, respectively, associated with the DY/arctic wolf haplotype. 

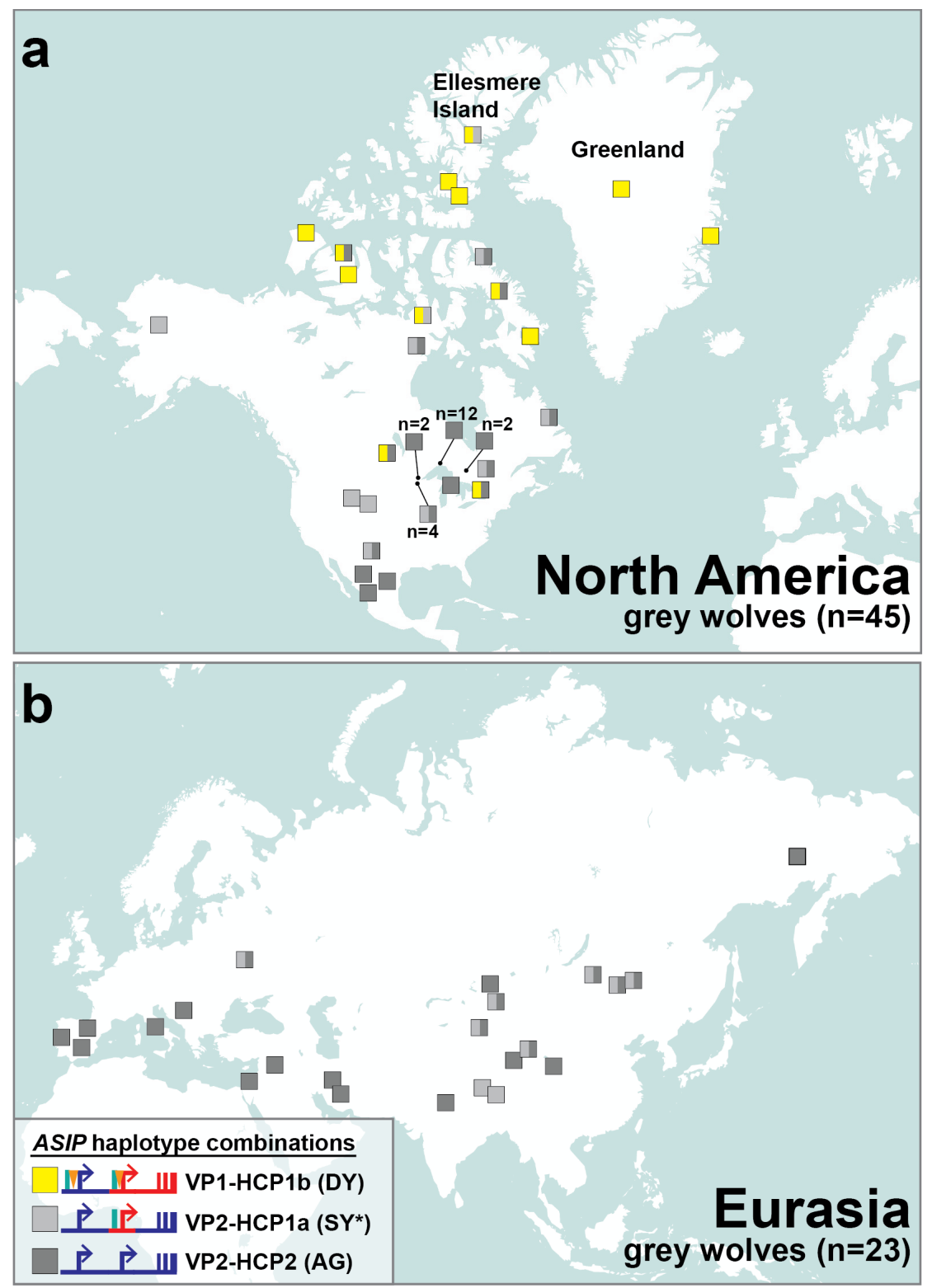

597 Extended Data Fig. 7: The distribution of $A$ SIP haplotypes in modern grey wolves. Modern

598 grey wolves from (a) North America $(n=45)$ or (b) Eurasia $(n=23)$ were genotyped for 5

599 structural variants and 6 SNVs using whole genome sequencing data. Each wolf, represented by

600 a colored box, is plotted information, summarized in the figure legend, is available in Extended

601 Data Fig. 8 and Supplementary Table 10. The asterisk indicates an SY-like haplotype without the

602 HCP1 insertion. 


\section{Inferred ancestral ASIP haplotypes \\ extinct Pleistocene canid Pleistocene grey wolf

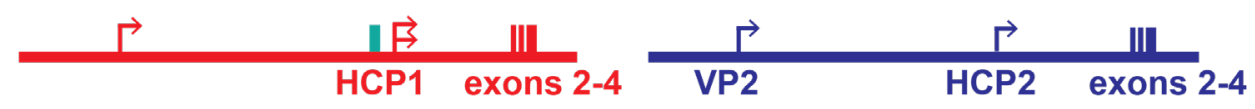

\section{Observed ASIP haplotypes}

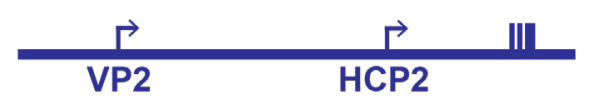

grey wolf

\& agouti dog

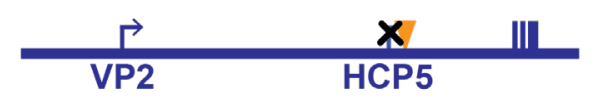

black back dog

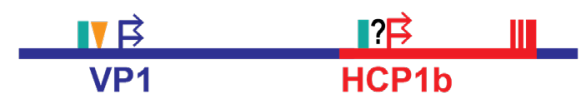

ancient grey wolf (i.e. Yana site)

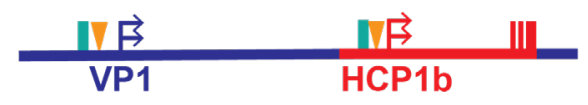

arctic grey wolf

\& dominant yellow dog

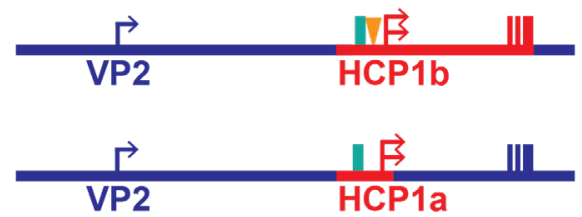

shaded yellow dog

603

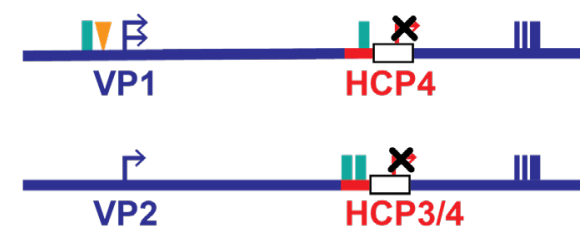

black saddle dog

black back dog

607 structural variants near the ventral (VP) and hair cycle (HCP) promoters are depicted as yellow

608 triangles (polynucleotide expansions), green bars (SINE insertions), and white bars (deletions).

609 Modified promoter activity is indicated by an X mark (no activity) or an additional arrow

610 (elevated expression), based on RNAseq (Extended Data Fig. 1) and/or inference from coat color

611 (Fig. 1, 3c). 
Extended Data Table 1. Segregation of modular promoter diplotypes with phenotype.

\begin{tabular}{|c|c|c|}
\hline Phenotype & Diplotype & Counts \\
\hline \multirow{2}{*}{ Dominant Yellow } & VP1-HCP1 / VP1,2-HCP1,3,4,5 & $113 / 114$ \\
& VP2-HCP1 / VP2-HCP1 & $1 * / 114$ \\
\hline \multirow{3}{*}{ Shaded Yellow } & VP2-HCP1 / VP2-HCP1,3,5 & $51 / 64$ \\
& VP2-HCP1 / VP1-HCP1 & $11^{* / 64}$ \\
& VP1-HCP1 / VP1-HCP1 & $2 * / 64$ \\
\hline Agouti & VP2-HCP2 / VP2-HCP2,3,5 & $46 / 46$ \\
\hline Black Saddle & VP1-HCP4 / VP1,2-HCP4,3 & $53 / 53$ \\
\hline Black Back & VP2-HCP3 / VP2-HCP3,4,5 & $89 / 89$ \\
\hline
\end{tabular}

614

615

* Dogs had MC1R based eumelanin masking pattern, which prevented reliable phenotype distinction between dominant yellow and shaded yellow.

Extended Data Table 2. SNVs distinguishing DY dogs and arctic wolves in the 64kb segment that contain the VP, HCP, and coding sequences.

620

\begin{tabular}{|c|c|c|c|c|c|}
\hline $\begin{array}{c}\text { Position } \\
\text { (Chr24, CF3) }\end{array}$ & $\begin{array}{c}\text { Ancestral } \\
\text { allele }\end{array}$ & $\begin{array}{c}\text { Derived } \\
\text { Allele }\end{array}$ & DY dog & Arctic wolf & Yana wolf \\
\hline $23,333,763$ & C & A & C & A & C \\
\hline $23,343,447$ & G & C & C & G & G \\
\hline $23,356,213$ & T & C & T & C & T \\
\hline $23,362,891$ & C & A & C & A & C \\
\hline $23,381,935$ & C & T & C & T & C \\
\hline $23,393,514$ & G & A & A & G & G \\
\hline
\end{tabular}

621

622

623

624 